\title{
Biotransformation of Ilaprazole in Human Liver Microsomes and Human: Role of CYP3A4 in llaprazole Clearance and Drug-Drug Interaction ${ }^{\text {[ }}$
}

\author{
Jie Pu, ${ }^{1}$ Fen Wang, Wei Tang, and Mingshe Zhu \\ DMPK Department ChemPartner, Shanghai, China (J.P., F.W., W.T.); School of Pharmacy, East China University of Science and \\ Technology, Shanghai, China (J.P.); and MassDefect Technologies, Princeton, New Jersey (M.Z.)
}

Received March 23, 2018; accepted July 9, 2018

\begin{abstract}
llaprazole is a new proton pump inhibitor and is currently marketed in China and South Korea for the treatment of gastric and duodenal ulcer. llaprazole has a favorable long half-life and minimal pharmacokinetic variability associated with CYP2C19 polymorphism. Sulfoxide oxidation of ilaprazole is catalyzed mainly by CYP3A4. Thus, it has been widely accepted that CYP3A4 plays a major role in the clearance of ilaprazole in humans. However, absorption, distribution, metabolism, and excretion data of radiolabeled ilaprazole in humans are not available. The primary goal of this study was to determine if sulfoxide oxidation is a major metabolic pathway of ilaprazole in humans. Metabolite profiles of ilaprazole, ilaprazole sulfide, and ilaprazole sulfone in human liver microsomes (HLMs) were characterized and quantitively analyzed by liquid chromatography (LC)/UV/high-resolution mass spectrometry (HRMS).

Moreover, metabolites of ilaprazole in human urine and feces were detected and identified by LC-HRMS. The results revealed that sulfoxide reduction to ilaprazole sulfide rather than sulfoxide oxidation was the major biotransformation pathway in HLMs. Sulfoxide reduction also occurred in HLMs without NADPH or in deactivated HLMs. Ilaprazole sulfide and its multiple oxidative metabolites were major drug-related components in human urine and feces, where there were no ilaprazole sulfone and its metabolites. A small amount of the parent drug was found in feces. Thus, we propose that nonenzymatic sulfoxide reduction rather than CYP3A4-medidated sulfoxide oxidation is the major metabolic clearance pathway of ilaprazole in humans. Consequently, it is predicted that ilaprazole has no significant drug-drug interaction via CYP3A4 inhibition or induction by a coadministered drug.
\end{abstract}

\section{Introduction}

Ilaprazole, the latest proton pump inhibitor (PPI), has been initially approved for the treatment of gastric and duodenal ulcers in China and South Korea, and has recently been approved for the treatment of erosive esophagitis in South Korea (Savarino et al., 2016). PPIs are the mainstay of the treatment of acid-related disease, such as gastro-esophageal reflux disease and peptic ulcer disease. The prevalence of gastro-esophageal reflux disease in Western countries ranges from $10 \%$ to $20 \%$ (Dent et al., 2005). The overall prevalence of peptic ulcer disease in China is $17.2 \%$ (Li et al., 2010). Administration of 10 and $20 \mathrm{mg}$ ilaprazole to gastroesophageal reflux disease patients has produced a greater and prolonged suppression of gastric $\mathrm{pH}$ than $20 \mathrm{mg}$ omeprazole (Periclou et al., 2000). The efficacy and safety of ilaprazole in the treatment of reflux esophagitis is similar to those of esomeprazole (Xue et al., 2016), while ilaprazole provides significantly better $\mathrm{pH}$ control over 24 hours compared with esomeprazole (Shin et al., 2014).

The pharmacodynamics and clinical outcomes of ilaprazole are associated with its improved drug metabolism and pharmacokinetics

${ }^{1}$ Current affiliation: School of Pharmacy and Pharmaceutical Sciences, SUNY at Buffalo, Buffalo, New York.

The authors declare that they have no conflict of interest.

https://doi.org/10.1124/dmd.118.081570.

S This article has supplemental material available at dmd.aspetjournals.org. proprieties compared with other PPIs. A majority of PPIs are metabolized mainly by CYP2C19 and partially by CYP3A4 (Klotz et al., 2004; Klotz, 2006; de Bortoli et al., 2013). The pharmacokinetics of PPIs such as omeprazole can be significantly affected by CYP2C19 polymorphism (Qiao et al., 2006; Hunfeld et al., 2008) and coadministered CYP2C19 inhibitors (Park et al., 2017). Ilaprazole overcomes the CYP2C19-related limitations of other PPIs. The plasma area-under-curve (AUC) and the peak concentration of ilaprazole are not affected by genetic polymorphisms of CYP2C19 (Li et al., 2008; Cho et al., 2012). Furthermore, the extension of plasma half-life of ilaprazole contributes to more rapid control of acid-related symptoms. The time to reach maximal plasma concentration for ilaprazole is between 3.4 and 3.7 hours and the elimination half-life ranges between 8.1 and 10.1 hours (de Bortoli et al., 2013), which are far better results than those of other currently available PPIs.

Sulfoxide oxidation to ilaprazole sulfone is observed in human liver microsome (HLM) incubation with ilaprazole, which is primarily catalyzed by CYP3A4 and partially by CYP3A5, while CYP1A2, 2A6, 2B6, 2C8, 2C9, 2C19, 2D6, and 2E1 have no sulfoxide oxidation activity (Seo et al., 2012). Thus, it has been widely accepted that CYP3A4 plays a major role in the clearance of ilaprazole in humans (Seo et al., 2012; de Bortoli et al., 2013; Shin et al., 2014; Savarino et al., 2016; Wang et al., 2016; Xuan et al., 2016). However, coadministered clarithromycin, a potent CYP3A4 inhibitor, does not elevate the

ABBREVIATIONS: HLM, human liver microsome; HRMS, high-resolution mass spectrometry; LC, liquid chromatography; MS, mass spectrometry; $\mathrm{m} / \mathrm{z}$, mass-to-charge ratio; P450, cytochrome P450; PPI, proton pump inhibitor. 
exposure of ilaprazole to human (Cao et al., 2012). The pharmacokinetics of ilaprazole was not affected by CYP3A phenotypes in 24 healthy subjects (Cao et al., 2015). On the other hand, major in vitro biotransformation reactions and metabolic clearance pathways of ilaprazole in humans remain unknown. We postulated that the lack of in vitro-in vivo correlation of the CYP3A-mediated metabolism of ilaprazole may be due to the fact that the sulfoxide oxidation of ilaprazole is not a major metabolic clearance pathway in humans.

To date, no absorption, distribution, metabolism and execration data of radiolabeled ilaprazole in animals and humans have been reported in the literature. Ilaprazole sulfone (M11) and ilaprazole sulfide (M12) were formed as significant drug-related components in HLMs (Fig. 1) (Seo et al., 2012). Four ilaprazole metabolites associated with sulfoxide reduction, including ilaprazole sulfide (M12) and three oxidative metabolites (M8, M6, and M20) of ilaprazole sulfide (Fig. 1), were found in human urine (Zhou et al., 2010). Ilaprazole sulfone and ilaprazole sulfide are also present in human and rat plasma (Myung et al., 1999; Zhou et al., 2009). Sulfoxide is the common moiety of the current PPIs, which undergoes cytochrome P450 (P450)-medicated sulfoxide oxidation and nonenzymatic sulfoxide reduction (de Bortoli et al., 2013). Unlike omeprazole, pantoprazole, and lansoprazole, which are cleared mainly via CYP2C19- and CYP3A4-medicated metabolism, rabeprazole undergoes extensive sulfoxide reduction in vitro (Ren et al., 2008) and in humans, while P450-mediated biotransformation pathways are not significant metabolic clearance pathways of rabeprazole in humans (Setoyama et al., 2006). Consequently, rabeprazole as a victim drug has no or minimal P450-mediated drug-drug interaction potentials (Yasuda et al., 1995; Shimizu et al., 2006). The current study was attempted to determine metabolic pathways of non-radiolabeled ilaprazole in HLMs and humans. The results from the study can help us to understand if CYP3A4-mediated sulfoxide oxidation to ilaprazole sulfone is the major metabolic clearance pathway in humans.

\section{Materials and Methods}

Chemicals. Ilaprazole was purchased from Cato Research Chemicals Inc. (Eugene, OR), and ilaprazole sulfide and ilaprazole sulfone were gifts from Livzon Pharmaceutical Group Inc (Guangdong, China). All solvents, including ethanol, acetonitrile, ethyl acetate, and water, were of high-performance liquid chromatography (LC) grade. Pooled HLMs were obtained from BD Biosciences (Woburn, MA).

Biotransformation of Ilaprazole in HLM Incubation. Ilaprazole (10 and $20 \mu \mathrm{M})$ was incubated with HLMs (1 mg protein/ml) and NADPH $(2 \mathrm{mM})$ in potassium phosphate buffer (100 mM, pH 7.4) with a total volume of $200 \mu \mathrm{l}$ at $37^{\circ} \mathrm{C}$ for 45 minutes. Incubation reactions were initiated by adding $100 \mu \mathrm{l}$ of NADPH after preincubation for 5 minutes at $37^{\circ} \mathrm{C}$ and stopped by adding $40 \mu \mathrm{l}$ of ammonium acetate $(\mathrm{pH} 9.5)$ and $600 \mu \mathrm{l}$ of ethyl acetate. Then, incubation mixtures were centrifuged at 14,000 rpm for 5 minutes. The upper organic phase was collected and evaporated to dryness under nitrogen stream. The remaining aqueous phase was mixed with $120 \mu \mathrm{l}$ of a methanol/water solution (7:3) followed by centrifugation. Resultant supernatants were evaporated to dryness under nitrogen stream. The dried samples were kept at $-20^{\circ} \mathrm{C}$ and reconstituted with a methanol/water (1:3) solution containing $5 \mathrm{mM}$ ammonium acetate prior to analysis by LC/mass spectrometry (MS). Use of liquid-liquid extraction instead of protein precipitation can effectively avoid the conversion of ilaprazole to ilaprazole sulfide during the precessing. The recovery of this method was tested to be very good. A control HLM incubation sample was prepared under the same incubation conditions except for the absence of NADPH. In addition, ilaprazole was incubated with inactivated HLMs (preheated at $80^{\circ} \mathrm{C}$ for 10 minutes) under the same conditions. The purpose of preheating the HLMs is to deactivate metabolizing enzyme activities.

\section{Proposed metabolic pathways of ilaprazole in HLM and human}

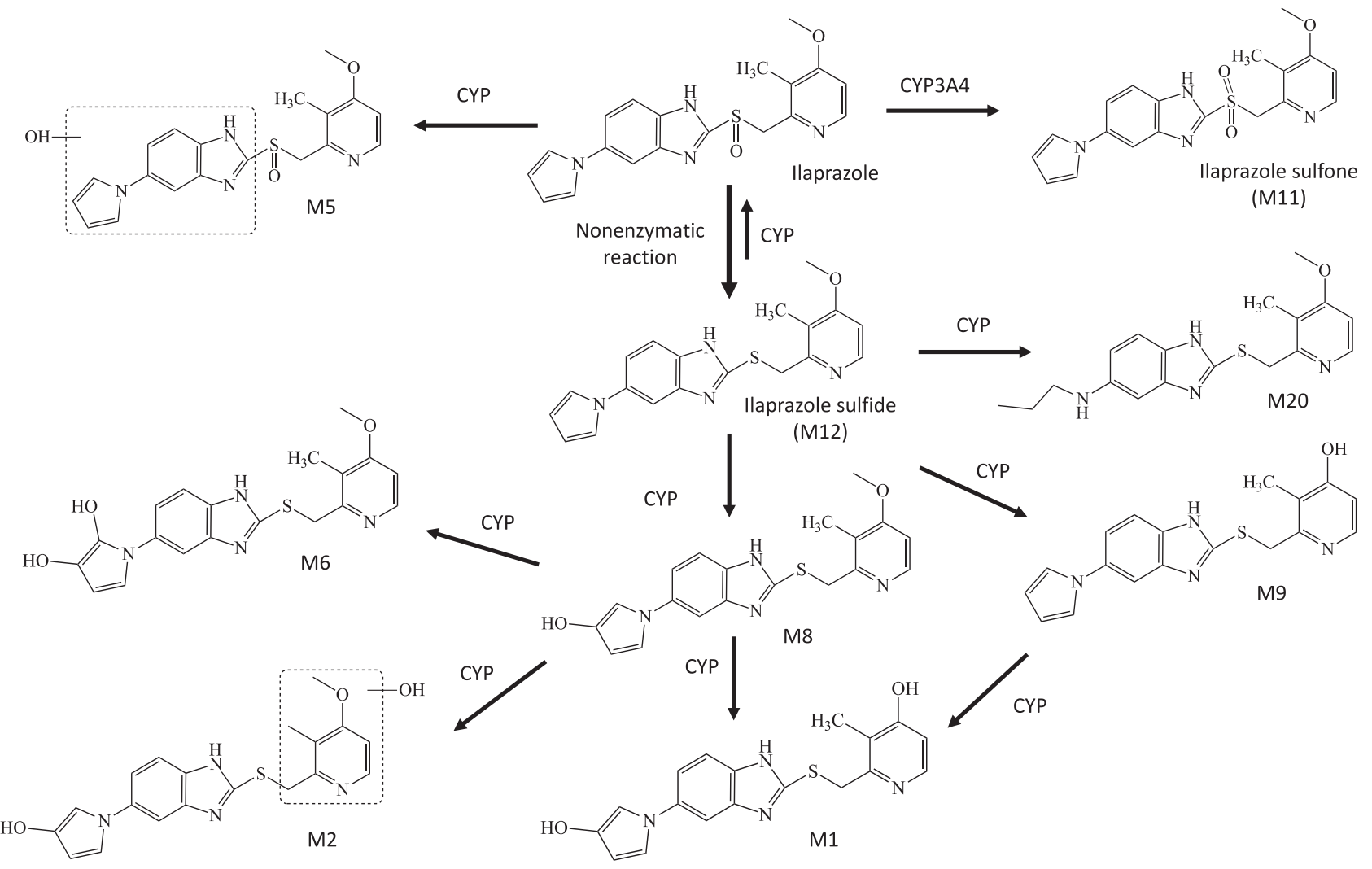

Fig. 1. Proposed major metabolic pathways of ilaprazole in HLMs and humans. 
Biotransformation of Ilaprazole Sulfide and Ilaprazole Sulfone in HLMs. Ilaprazole sulfide and ilaprazole sulfone $(20 \mu \mathrm{M})$ were incubated separately with HLMs under the same conditions followed by the same sample treatment processes described previously. Control incubation samples (without NADPH) were also generated in the same way.

Determination of Metabolic Stability of Ilaprazole, Ilaprazole Sulfide, and Ilaprazole Sulfone in HLMs. Ilaprazole, ilaprazole sulfide, and ilaprazole sulfone $(1 \mu \mathrm{M})$ were incubated separately with HLMs $(0.75 \mathrm{mg}$ protein $/ \mathrm{ml})$ and NADPH $(2 \mathrm{mM})$ in potassium phosphate buffer $(100 \mathrm{mM}, \mathrm{pH} 7.4,1.0$ EDTA) with a total volume of $200 \mu \mathrm{l}$ at $37^{\circ} \mathrm{C}$ for $0,5,15,30$, and 45 minutes. The incubation reactions were initiated by adding $30 \mu \mathrm{l}$ of NADPH after preincubation for 5 minutes and stopped by adding $270 \mu \mathrm{l}$ of methanol containing omeprazole $(1 \mu \mathrm{M})$. Omeprazole was used as an interval standard for quantitative analysis by LC/MS. After centrifugation at 14,000 rpm for 5 minutes, $100 \mu \mathrm{l}$ of supernatant was mixed with $100 \mu \mathrm{l}$ of water followed by analysis of remaining ilaprazole, ilaprazole sulfide, or ilaprazole sulfone by LC/MS.

Determination of Ilaprazole Stability in Human Plasma. Ilaprazole $(4 \mu \mathrm{M})$ was incubated with pooled human plasma at $37^{\circ} \mathrm{C}$ for $0,5,15,45,90,120,180$, 240 , and 300 minutes. The incubation was initiated by adding $10 \mu \mathrm{l}$ of prewarmed ilaprazole solution $(0.5 \%$ bovine serum albumin) into $90 \mu \mathrm{l}$ prewarmed plasma and stopped by adding $270 \mu \mathrm{l}$ of methanol containing omeprazole. The incubation mixtures were centrifuged at $14,000 \mathrm{rpm}$ for 5 minutes and $50 \mu \mathrm{l}$ of supernatants was mixed with $100 \mu \mathrm{l}$ of water for quantitative analysis of the remaining ilaprazole using LC/MS.

Collection and Treatment of Human Urine and Feces after Oral Administration of Ilaprazole. Two healthy Chinese volunteers (subject A: female, 22 years old; subject B: female, 54 years old) received a single dose of ilaprazole $(10 \mathrm{mg})$ with an empty stomach. Urinary samples from the two subjects were collected at 0-24 and 24-48 hours after dosing. Fecal samples were collected from subject $A$ after time periods of $0-4,4-33$, and 33-48.5 hours and from subject $B$ after time periods of 0-24 and 24-49 hours. In addition, control urine and feces samples were collected from the two subjects prior to dosing. All urine and feces collected were stored at approximately $-20^{\circ} \mathrm{C}$. In the urine sample process, $1200 \mu \mathrm{l}$ methanol was mixed with $400 \mu \mathrm{l}$ urinary samples. After centrifugation at $14,000 \mathrm{rpm}$ for 10 minutes, supernatant was evaporated to dryness under nitrogen stream and then reconstituted with $70 \mu \mathrm{l}$ of the methanol/water solution prior to LC/MS analysis. In the feces sample process, each sample was weighted around 330-350 mg. The same volume of water and twice the volume of methanol were added into the feces samples, followed by vortexing. For example, if the feces sample was $350 \mathrm{mg}$, then $350 \mu \mathrm{l}$ of water and $700 \mu \mathrm{l}$ of methanol were added. After centrifugation at $14,000 \mathrm{rpm}$ for 10 minutes, the supernatant was kept and the remaining precipitation was extracted twice with the water/methanol solution. All supernatants were combined and evaporated to dryness under nitrogen stream and reconstituted with the methanol/water solution prior to LC/MS analysis.

Metabolite Detection and Characterization by LC/UV/High-Resolution MS. LC/high-resolution MS (HRMS) consisted of an ACQUITY UPLC System (Waters, Milford, MA) and a G2-S Q-Tof mass spectrometer (Waters). The ACQUITY UPLC System was equipped with an XBridge ACQUITY UPLC BEH C18 column $(2.1 \times 50 \mathrm{~mm}, 1.7 \mu \mathrm{m}$; Waters $)$, a photodiode array detector, and an autosampler (Thermo Fisher Scientific, Waltham, MA). The LC/UV/HRMS system was used for detection, quantitative estimation, and/or structural identification of metabolites of ilaprazole, ilaprazole sulfide, and ilaprazole sulfone in HLMs as well as ilaprazole metabolites in human urine and feces. Relative concentrations of ilaprazole metabolites in HLM incubation were determined by comparing UV peak intensity of metabolites. In addition, LC/HRMS was employed to measure relative concentrations of testing compounds in the HLM metabolic and human plasma stability experiments.

For detection and characterization of metabolites in HLMs and quantitative analysis of testing compounds in HLM metabolic and human plasma stability studies, an LC gradient system consisting of solvent A ( $5 \mathrm{mM}$ ammonium acetate in water) and solvent B (methanol) was set up as follows. A linear gradient of solvent B started at $2 \%$ for 1.5 minutes, and then went to $95 \%$ from 7 minutes for 1.5 minutes. After that, it dropped back to $2 \%$ at 8.6 minutes for 1.4 minutes. The total run time was 10 minutes and the flow rate was $400 \mu \mathrm{l} / \mathrm{min}$. An on-line UV detector was set up with absorbance at 230-240 nm. For profiling and identification of metabolites in human urine and feces, the same solvents (A) and (B) as described previously were employed and an LC gradient system was set up as follows: A linear gradient of solvent B started at $2 \%$, then went to
$5 \%$ from 3 minutes. After being raised to $40 \%$ at 11 minutes and kept for 6 minutes, the gradient went to $90 \%$ from 17 to 20 minutes, and then finally returned to $2 \%$. All compounds were detected by UV absorbance at 230-240 nm. The total run time was 22 minutes and the flow rate was $400 \mu \mathrm{l} / \mathrm{min}$. HRMS was set up in positive ion mode with capillary of $2.00 \mathrm{kV}$, source temperature of $120^{\circ} \mathrm{C}$, desolvation temperature of $500^{\circ} \mathrm{C}$, cone gas of $50 \mathrm{l} / \mathrm{h}$, and desolvation gas of $800 \mathrm{l} / \mathrm{h}$. Fragment and product ion spectra of metabolites were recorded by simultaneous acquisition of exact mass at high and low collision energy $\left(\mathrm{MS}^{\mathrm{E}}\right)$ (20-40 V rump collision energy) and targeted MS/MS acquisition, respectively. Comprehensive detection of metabolites was carried out by processing full MS and fragment ion or product spectral data sets using mass defect filter, extracted ion chromatographic analysis, and product ion filter (Zhu et al., 2006; Ruan et al., 2008; Ma and Chowdhury, 2013). Determination of metabolite structures was accomplished based on spectral interpretation and/or comparisons with synthetic standards (M11 and M12) as well as previously reported metabolites (M6, M8, and M20), whose structures were determined by nuclear magnetic resonance (Zhou et al., 2010).

Metabolite Detection and Characterization by LC/Ion Trap MS. A LC/ion trap MS system that consisted of an Accela high-performance liquid chromatography system equipped with an Xbridge C18 column $(2.1 \times 150 \mathrm{~mm}$, $5 \mu \mathrm{m}$ ) (Waters), a photodiode array detector, and a LTQ XL ion trap mass spectrometer (Thermo Fisher Scientific) was employed for profiling and quantitative analysis of ilaprazole metabolites, mainly sulfide, in HLM incubations under various conditions. Relative concentrations of ilaprazole metabolites in HLM incubation were accomplished by direct analysis using LC/UV. The photodiode array detector was set up with UV absorbance at 230-240 nm. A linear highperformance liquid chromatography gradient system was developed with mobile phases consisting of solvent $\mathrm{A}$ ( $5 \mathrm{mM}$ ammonium acetate in water) and solvent $\mathrm{B}$ (methanol). A linear gradient of solvent B start at $2 \%$, and then went to $5 \%$ from 0.34 minutes. After being raised to $40 \%$ at 9 minutes and kept for 5.5 minutes, the gradient went to $90 \%$ from 14.5 to 18 minutes, and then finally returned to $2 \%$. The total run time was 22 minutes and the flow rate was $300 \mu \mathrm{l} / \mathrm{min}$. The mass spectrometer was operating in the positive electrospray ionization mode. The capillary temperature was at $275^{\circ} \mathrm{C}$ and the capillary voltage was at $38 \mathrm{~V}$. Product ion spectra of metabolites were acquired using a data-dependent scanning method.

\section{Results}

Metabolite Profiles of Ilaprazole in HLMs. The LC/HRMS analysis of HLM incubation samples with ilaprazole (10 and $20 \mu \mathrm{M}$, 45 minutes) found a total of 11 ilaprazole metabolites: M1, M2, and M4-M12 (Fig. 2B and Table 1). The proposed structures of these metabolites and their accurate product ion spectra or fragment ion spectra are displayed in Figs. 1 and 3 and Supplemental Fig. 1 and 2. Based on quantitative estimation using LC/UV (Fig. 2A), it was determined that about $30 \%$ of ilaprazole was converted to these metabolites, mainly, M4, M5, M7-M9, M11, and M12. M11 had a UV peak area 3-fold or greater than those of the other metabolites. M4 and M5 were overlapped in the LC/UV (Fig. 2A) and LC/MS profile (Fig. 2B). Two minor metabolites, M1 and M6, were only detected by LC/MS (Fig. 2B).

Metabolite Profiles of Ilaprazole Sulfide in HLMs. Ilaprazole sulfide (M12) $(20 \mu \mathrm{M})$ was incubated in HLMs for 45 minutes, followed by analysis using LC/UV/HRMS. As shown in Fig. 2, C and D and Table 1, ilaprazole sulfide generated 12 oxidative metabolites in HLMs. All of the ilaprazole metabolites were found in the HLM incubation of ilaprazole sulfide. Additionally, the ilaprazole sulfide metabolites, M13 and M15, were not observed in the HLM incubation with ilaprazole. The proposed structures and accurate mass spectral data of M13 and M15 are presented in Supplemental Fig. 1. An LC/UV metabolite profile of the same incubation sample showed that M2, M6, M8, M9, and ilaprazole were major metabolites of ilaprazole sulfide in HLMs (Fig. 2C).

Metabolite Profiles of Ilaprazole Sulfone in HLMs. The LC/HRMS analysis of the HLM incubation sample with ilaprazole sulfone (20 $\mu \mathrm{M}, 45$ minutes) revealed four metabolites: M16-M19 

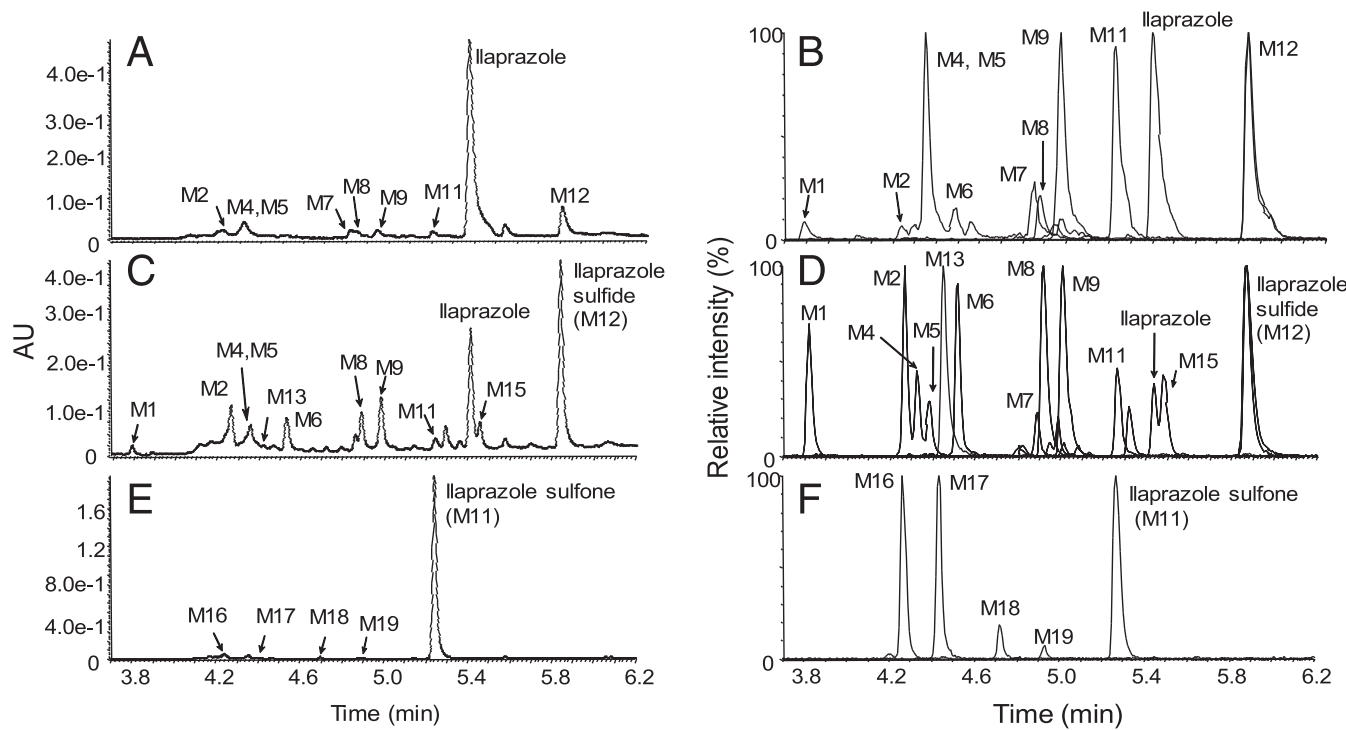

Fig. 2. LC/UV/MS analysis of metabolites of ilaprazole, ilaprazole sulfide, and ilaprazole sulfone in HLMs (20 $\mu$ M for 45 minutes). LC/UV profiles of metabolites of ilaprazole (A), ilaprazole sulfide (C), and ilaprazole sulfone (E). Extracted ion chromatograms of metabolites of ilaprazole (B), ilaprazole sulfide (D), and ilaprazole sulfone (F). The similar metabolite profile of ilaprazole was generated with HLM incubation of ilapraozle at $10 \mu \mathrm{M}$ (data not shown).

(Fig. 2F). The concentrations of these metabolites were very low, as shown in the LC/UV profile of the same incubation sample (Fig. 2E). The proposed structures of these metabolites and their product spectral data are displayed in Supplemental Fig. 1. M17 was identified as O-demethyl ilaprazole sulfone. M15, M17, and M18 were characterized as monooxidation metabolites of ilaprazole sulfone. Interestingly, these ilaprazole sulfone metabolites were not observed in the HLM incubations of ilaprazole or ilaprazole sulfide (Table 1).

Structural Elucidation of HLM Metabolites of Ilaprazole and Ilaprazole Sulfide. M12 and M11 were determined to be ilaprazole sulfide and ilaprazole sulfone (Fig. 1), respectively, by comparing their LC/MS data with ilaprazole sulfide and ilaprazole sulfone standards. As shown in the product or fragment ion spectra of ilaprazole (Supplemental
Fig. 1), ilaprazole, ilaprazole sulfide, and ilaprazole sulfone (Fig. 3) shared the same major fragmentation pathways: the cleavage of the C-S bond between the benzimidazole and sulfur atom leading to a substituted benzimidazole ion [such as an ion at mass-to-charge ratio $(\mathrm{m} / \mathrm{z}) 184.0882$ from M11 in Fig. 3] and an ion corresponding to the $\mathrm{S}-\mathrm{CH}_{2}$ containing pyridine derivative (such as an ion at $\mathrm{m} / \mathrm{z} 184.0447$ from ilaprazole in Supplemental Fig. 1). Ilaprazole sulfide has a unique fragmentation associated with the sulfide structure: a loss of SH from the protonated molecule at $\mathrm{m} / \mathrm{z} 351.1281$ leading to a product ion at $\mathrm{m} / \mathrm{z} 318.1480$ (Fig. 3). Those diagnostic fragmentations were used to facilitate spectral interpretation of unknown metabolites from ilaprazole, ilaprazole sulfide, and ilaprazole sulfone. M5 had a protonated molecule at $\mathrm{m} / \mathrm{z}$ 383.1173, which was 16 Da greater than ilaprazole. The cleavage of the benzimidazole and

TABLE 1

Summary of metabolites found in human urine and feces following an oral dose of ilaprazole as well as observed in HLM incubations with ilaprazole, ilaprazole sulfide, and ilaprazole sulfone

\begin{tabular}{|c|c|c|c|c|c|c|c|c|}
\hline \multirow{2}{*}{ Metabolite } & \multirow{2}{*}[\mathrm{M}+\mathrm{H}]{$^{+}$} & \multirow{2}{*}{ Identity $^{a}$} & \multirow{2}{*}{ Product ion or fragment ion ${ }^{b}$} & \multicolumn{3}{|c|}{ HLM incubation } & \multicolumn{2}{|c|}{ Human } \\
\hline & & & & Ilaprazole & Ilaprazole sulfide & Ilaprazole sulfone & Urine & Feces \\
\hline P (Ilaprazole) & 367.1234 & $\mathrm{P}(\mathrm{M} 12+\mathrm{O})$ & $184,166,137,136,122,106$ & + & + & & & + \\
\hline M1 & 353.1073 & $\mathrm{P}-\mathrm{CH}_{2}\left(\mathrm{M} 12-\mathrm{CH}_{2}+\mathrm{O}\right)$ & $320,200,154,123,122$ & + & + & & + & \\
\hline M2 & 383.1193 & $\mathrm{P}+\mathrm{O}(\mathrm{M} 12+2 \mathrm{O})$ & $365,200,184,166,152$ & + & + & & + & + \\
\hline $\mathrm{M} 4^{c}$ & 383.1151 & $\mathrm{P}+\mathrm{O}(\mathrm{M} 12+2 \mathrm{O})$ & & + & + & & & \\
\hline M5 & 383.1173 & $\mathrm{P}+\mathrm{O}(\mathrm{M} 12+2 \mathrm{O})$ & $365,200,184,166,137,122,106$ & + & + & & + & \\
\hline M6 & 383.1158 & $\mathrm{P}+\mathrm{O}(\mathrm{M} 12+2 \mathrm{O})$ & $350,216,168,137,136,106$ & + & + & & + & + \\
\hline $\mathrm{M} 7^{c}$ & 383.1158 & $\mathrm{P}+\mathrm{O}(\mathrm{M} 12+2 \mathrm{O})$ & & + & + & & & \\
\hline M8 & 367.1227 & $\mathrm{P}-\mathrm{O}+\mathrm{O}(\mathrm{M} 12+\mathrm{O})$ & $334,200,168,137,136,106$ & + & + & & + & \\
\hline M9 & 337.1124 & $\mathrm{P}-\mathrm{O}-\mathrm{CH}_{2}\left(\mathrm{M} 12-\mathrm{CH}_{2}\right)$ & $304,287,184,154,122$ & + & + & & + & + \\
\hline M10 & 399.1528 & $\mathrm{P}-\mathrm{O}+3 \mathrm{O}(\mathrm{M} 12+3 \mathrm{O})$ & $366,232,168,137,136,122,106$ & + & + & & & \\
\hline M11 (Ilaprazole sulfone) & 383.1178 & $\mathrm{P}+\mathrm{O}(\mathrm{M} 12+2 \mathrm{O})$ & $200,184,137,136,122,106$ & + & + & + & & \\
\hline M12 (Ilaprazole sulfide) & 351.1266 & $\mathrm{P}-\mathrm{O}$ & $318,184,168,137,136,122,106$ & + & + & & + & + \\
\hline M13 & 301.1125 & $\mathrm{M} 12-\mathrm{C}_{4} \mathrm{H}_{2}$ & $268,168,137,134,122,106$ & & + & & & \\
\hline $\mathrm{M} 15^{c}$ & 367.123 & $\mathrm{M} 12+\mathrm{O}$ & $349,317,184,152$ & & + & & & \\
\hline M16 & 399.1128 & $\mathrm{M} 11+\mathrm{O}$ & $200,136,122,106$ & & & + & & \\
\hline M17 & 369.1018 & $\mathrm{M} 11-\mathrm{CH}_{2}$ & $186,184,122$ & & & + & & \\
\hline M18 & 399.1136 & $\mathrm{M} 11+\mathrm{O}$ & $381,216,184$ & & & + & & \\
\hline M19 & 399.1147 & $\mathrm{M} 11+\mathrm{O}$ & $216,184,152$ & & & + & & \\
\hline M20 & 343.124 & $\mathrm{P}-\mathrm{O}-\mathrm{C}+4 \mathrm{H}(\mathrm{M} 12-\mathrm{C}+4 \mathrm{H})$ & $310,176,168,137,106$ & & & & + & \\
\hline
\end{tabular}

${ }^{a}$ Identified or proposed metabolite structures are shown in Fig. 1 and Supplemental Figs. 1 and 2.

${ }^{b}$ Accurate mass product ion spectra (M1, M2, M4-M12, and M15) and fragment ion spectra (M13 and M16-M2) were acquired, which are displayed in Fig. 3 and Supplemental Figs. 1 and 2. ${ }^{c}$ No reliable or interpretable fragment ion spectral data were acquired. 

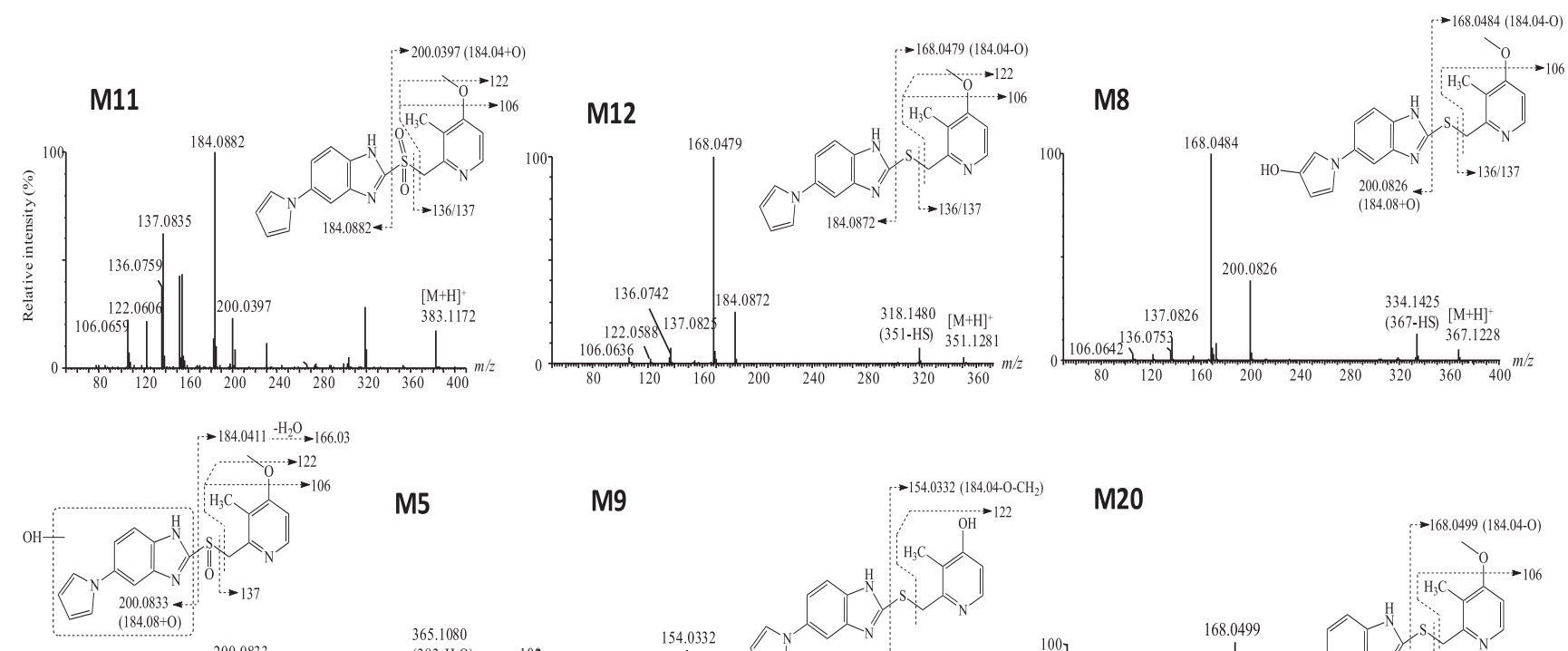

M5
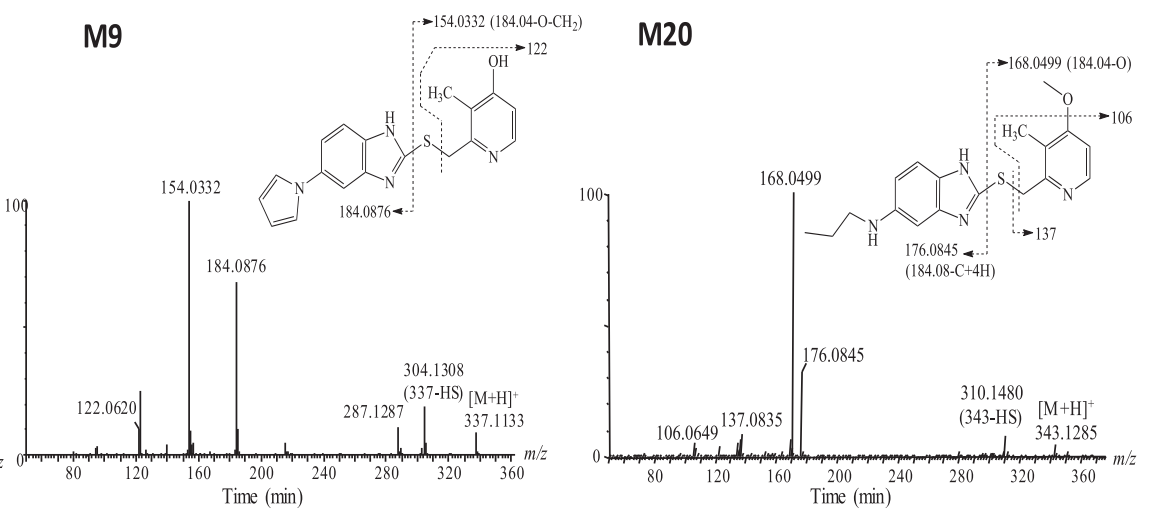

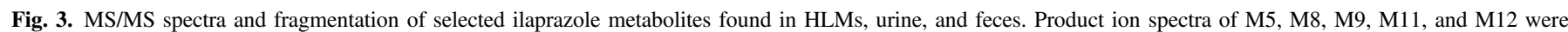

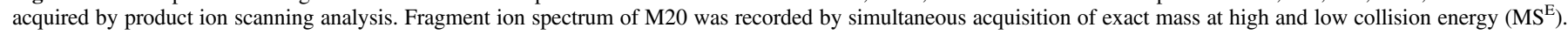

sulfur atom led to an ion at $\mathrm{m} / \mathrm{z}, 200.0833$ (substituted benzimidazole $+\mathrm{O}$ ) and $m / z, 184.0411$ ( $\mathrm{S}-\mathrm{CH}_{2}$ containing pyridine derivative), suggesting the oxygen atom was added in the substituted benzimidazole moiety. Thus, M5 was determined to be a monooxidation product of ilaprazole (see Fig. 3 as well as Fig. 5). Most likely, the oxygen atom was attached to one of the nitrogen atoms since a product ion at $\mathrm{m} / \mathrm{z} 365.1086$ corresponding to a loss of water from the protonated molecule was very significant. M4 and M7 had the same protonated molecule corresponding to the addition of an oxygen atom to ilaprazole such that M4 and M7 were determined to be monooxidation products of ilaprazole (Table 1). However, since both metabolites had no high-quality fragment ion spectral data, the defined structures of M4 and M7 remain to be determined.

M8 and M6 were assigned as monohydroxyl and dihydroxyl ilaprazole sulfide, respectively, in which hydroxyl groups are on the pyrrole ring, The product ion spectra of M8 (Fig. 3) and M6 (Supplemental Fig. 1) were almost identical to those of the same metabolites reported in the literature, in which structures of these two metabolites were determined by nuclear magnetic resonance analysis (Zhou et al., 2010). M9 had a protonated molecule at $\mathrm{m} / \mathrm{z}$ 337.1133, which was 14 Da smaller than that of ilaprazole sulfide. Both shared the same fragmentation pattern (Fig. 3). Thus, M9 was characterized as dimethyl ilaprazole sulfide. In the same manner, M1 was assigned to be desmethyl M8. M13 was a product formed via dealkylation of the pyrrole ring of ilaprazole (Supplemental Fig. 1). M2 was characterized as a monooxidation product of M8 (Fig. 3).

Role of HLM Enzymes in Sulfoxide Reduction of Ilaprazole. To determine the roles of $\mathrm{P} 450$ and other metabolizing enzymes in sulfoxide reduction of ilaprazole, ilaprazole was incubated with HLMs under various conductions, followed by quantitative analysis of ilaprazole sulfide by LC/UV/MS (Fig. 4). Compared with the HLM incubation control sample (without zero time) (Fig. 4A), HLM incubation of ilaprazole (10 $\mu \mathrm{M}, 45$ minutes) with NADPH generated ilaprazole sulfide (M12), ilaprazole sulfone (M11), M9, and M8 (Fig. 4C), among which M12 was the major metabolite. The HLM incubation of ilaprazole in the absence of NADPH also led to the formation of M12, but not other oxidative metabolites, M11, M8, and M9 (Fig. 4B), indicating that P450 was responsible for the formation of M12, M8, and M9, but not sulfoxide reduction of ilaprazole. Moreover, incubation of ilaprazole with deactivated HLMs (preheated at $80^{\circ} \mathrm{C}$ for 10 minutes) and NADPH generated M12 but not M11, M8, and M9 (Fig. 4D). Relative amounts of M12 formed in the incubations with HLMs and deactivated HLMs were comparable (Fig. 4, C and D). These observations further indicate that sulfoxide reduction to ilaprazole sulfide was mainly a chemical reaction with no involvement of P450 enzymes or significant contributions by other metabolizing enzymes in HLMs.

Metabolic Stability of Ilaprazole, Ilaprazole Sulfide, and Ilaprazole Sulfone in HLMs. Metabolic stability of ilaprazole, ilaprazole sulfide, and ilaprazole sulfone $(1 \mu \mathrm{M})$ was determined by measuring their disappearances in HLM incubations for up to 45 minutes using LC/MS. As shown in Fig. 5A, the half-life values of the disappearances for ilaprazole, ilaprazole sulfide, and ilaprazole sulfone were 33.8, 8.0, and 143.5 minutes, respectively, indicating that metabolism of ilaprazole sulfide was much faster than ilaprazole, while ilaprazole sulfone was metabolically stable in HLMs.

Stability of Ilaprazole in Human Plasma. Stability of ilaprazole (4 $\mu \mathrm{M})$ in human plasma was determined by measuring the relative concentrations of ilaprazole at multiple time points using LC/MS. As shown in Fig. 5B, the disappearance of ilaprazole after incubation for 120 minutes was minimal, indicating ilaprazole was very stable in human plasma.

Detection and Structural Characterization of Ilaprazole Metabolites in Human Urine and Feces. Human urine and feces samples collected from two healthy female subjects after oral administration of 

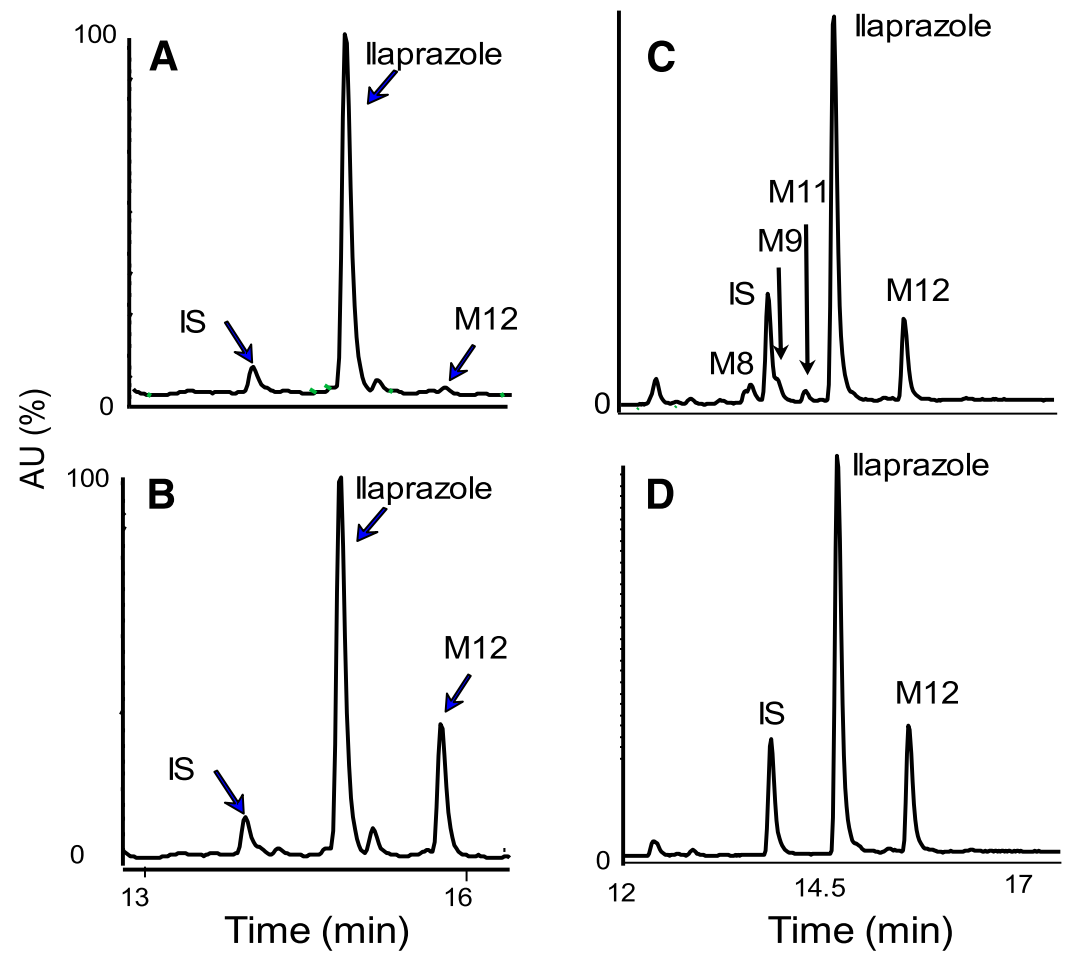

Fig. 4. LC/UV profiles of incubation samples of ilaprazole $(10 \mu \mathrm{M})$ with HLMs under various conductions. (A) Ilaprazole mixed with HLMs and NADPH without incubation (negative control). (B) Incubation of ilaprazole in HLMs without NADPH for 45 minutes. (C) Incubation of ilaprazole in HLMs with NADPH for 45 minutes. (D) Incubation of ilaprazole with deactivated HLMs and NADPH for 45 minutes.

$10 \mathrm{mg}$ ilaprazole were subjected to analysis by LC/UV/HRMS. Detection of potential ilaprazole metabolites was performed by searching for metabolites observed in the HLM incubations with ilaprazole, ilaprazole sulfide, and ilaprazole sulfone (Table 1). In addition, predicted

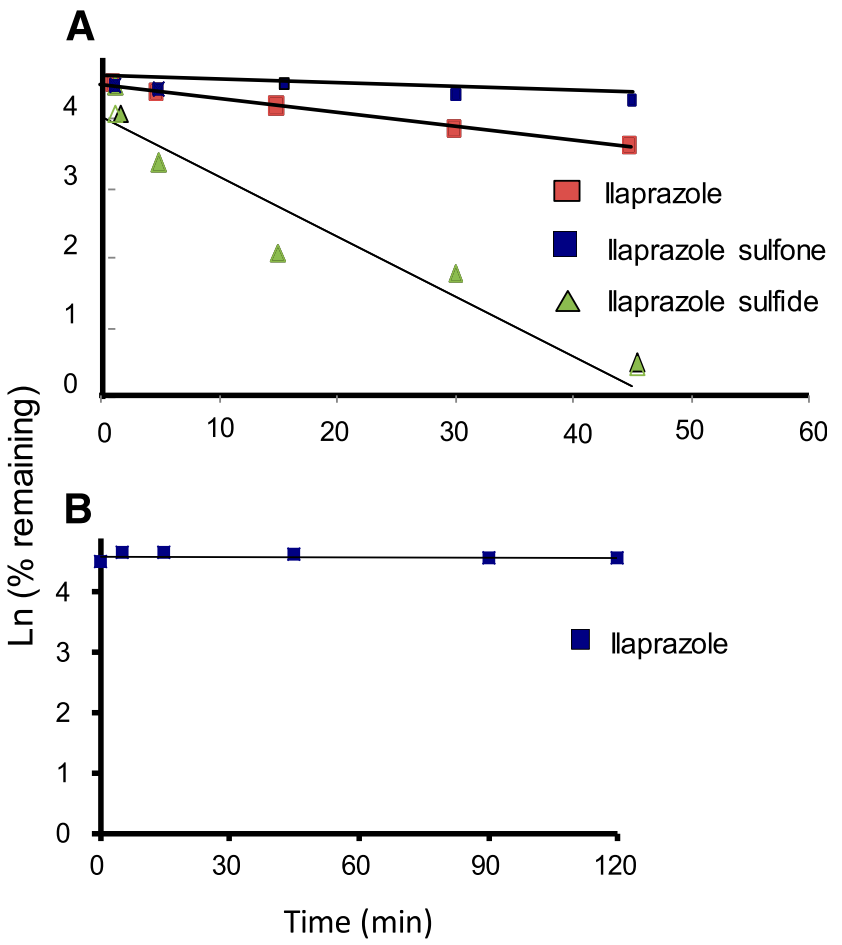

Fig. 5. (A) Disappearance of ilaprazole, ilaprazole sulfide, and ilaprazole sulfone in HLM incubation with NADPH. (B) Disappearance of ilaprazole in incubation with human. Individual test compounds were incubated in HLMs or human plasma at $37^{\circ} \mathrm{C}$ in triplicate. Remaining test compounds in the incubations were measured by LC/HRMS. common glucuronide and sulfate conjugates of all metabolites listed in Table 1 were searched. Furthermore, mass defect and product ion filters were employed to process full MS and fragment ion LC/MS data sets to discover unpredictable metabolites of ilaprazole and ilaprazole sulfone. As a result, eight significant drug-related components, M1, M2, M5, M6, M8, M9, M12, and M20, were detected and structurally characterized in the human urine (Fig. 6, B and D). Four metabolites, M2, M6, M9, and M12, were found in the human feces (Fig. 6, A and C). The ilaprazole metabolite profiles in urine and feces from the two subjects were the same. All of the metabolites found in human urine and feces except for M5 were either ilaprazole sulfide (M12) or metabolites of ilaprazole sulfide. In addition, all of the in vivo human metabolites except for M20 were formed in the HLM incubation with ilaprazole (Table 1). M20 was a metabolite derived from $N$-dealkylation of the pyrrole ring of ilaprazole sulfide (Fig. 3). M13, a downstream metabolite of M20, was seen in the HLM incubation with ilaprazole sulfide (Fig. 2D). It is worth noting that neither ilaprazole sulfone (M11) nor its metabolites formed in HLMs (M16-M19) or predicted by common biotransformation pathways were detected in human urine or feces (Table 1). A small amount of ilaprazole was found in human feces, but not in human urine (Fig. 6).

\section{Discussion}

It has been widely accepted that CYP3A4-mediated metabolism plays a major role in ilaprazole clearance in humans (Seo et al., 2012; de Bortoli et al., 2013; Shin et al., 2014; Savarino et al., 2016; Wang et al., 2016; Xuan et al., 2016). The key experimental evidence to support this consideration is that sulfoxide oxidation to ilaprazole sulfone is observed in HLMs and CYP3A4 mainly catalyzes the formation of ilaprazole sulfone (Seo et al., 2012). Inhibition or induction of CYP3A4 by a coadministered drug may lead to very significant changes (5- to 10-fold) in exposure of a victim drug that is mainly cleaned by a CYP3A4mediated metabolic pathway in humans (Hisaka et al., 2009; Fenneteau et al., 2010). Thus, good understanding of the drug-drug interaction potential of ilaprazole as a victim of a CYP3A4 inhibitor or inductor is 

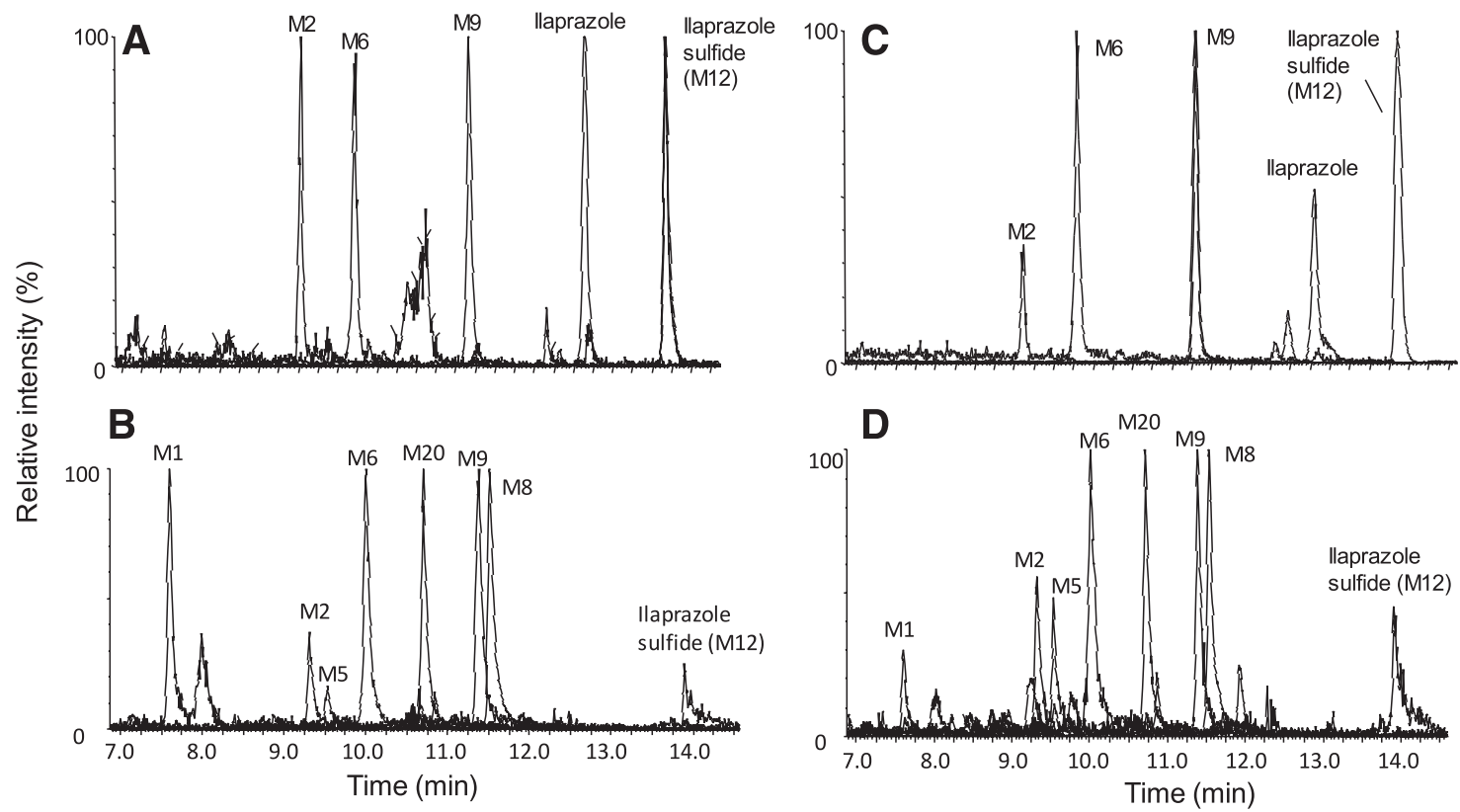

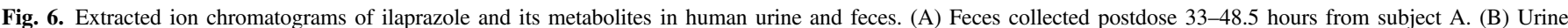
collected postdose 0-48 hours from subject A; (C) Feces collected postdose 24-49 hours from subject B; (D) Urine collected postdose 0-48 hours from subject B.

very important in the clinical use of ilaprazole. Omeprazole, lansoprazole, and esomeprazole are metabolized mainly by CYPC19 and partially by CYP3A. Exposures of these PPIs to humans are increased by 1.3 - to 2-fold by administered clarithromycin, a potent CYP3A inhibitor (Mainz et al., 2002; Ushiama et al., 2002; Calabresi et al., 2004). In contrast, rabeprazole is mainly cleaned via nonenzymatic sulfoxide reduction (Setoyama et al., 2006). Therefore, the exposure of rabeprazole to humans is not elevated when clarithromycin is coadministered (Yasuda et al., 1995; Shimizu et al., 2006). We speculated that sulfoxide oxidation to ilaprazole sulfide instead of CYP3A4mediated nonenzymetic sulfoxide reduction to ilaprazole sulfone is the major clearance pathway of ilaprazole in humans, which would explain the observation that the inhibition of CYP3A4 by clarithromycin has not elevated the exposure of ilaprazole to humans (Cao et al., 2012).

The primary goal of this study was to determine if sulfoxide oxidation is a major metabolic clearance pathway of ilaprazole in humans. LC/HRMS analysis of ilaprazole metabolites in HLMs (Fig. 2B) releveled that ilaprazole underwent three primary metabolic pathways in HLMs: sulfoxide reduction to ilaprazole sulfide; monooxidation to M4, M5, and M7; and sulfoxide oxidation to ilaprazole sulfone (Figs. 1 and 2; Table 1). Quantitative analysis of ilaprazole metabolites by LC/UV showed that the formation of ilaprazole sulfone was a relatively minor metabolic pathway compared with the sulfoxide reduction of ilaprazole in HLMs (Fig. 2, A and B). Moreover, LC/HRMS analysis of human urine and feces did not find ilaprazole sulfone and its metabolites (M16-M19) observed in HLMs (Table 1) as well as potential glucuronide and sulfate conjugates of those ilaprazole sulfone-related metabolites (Fig. 6; Table 1), although ilaprazole sulfone was previously found in human plasma (Zhou et al., 2009; Cao et al., 2015). Most likely, a small amount of ilaprazole sulfone is formed in human liver via CYP3A4mediated sulfoxide oxidation and then slowly converted to a few oxidative metabolites (M16-M19) (Fig. 2, E and F; Fig. 5A). Ilaprazole sulfone and its metabolites (M16-M19) could be directly eliminated via kidney and/or liver. However, due to very low abundances in feces and/or urine, LC/HRMS analysis was not able to find them. Collectively, the results from the in vitro and in vivo biotransformation experiments provide strong evidence that CYP3A4-mediated sulfoxide oxidation to ilaprazole sulfone is not a major metabolic clearance pathway in humans.

Although ilaprazole sulfide was previously found in HLM incubation (Seo et al., 2012) and human plasma and urine (Zhou et al., 2009, 2010), the important role of sulfoxide reduction to the metabolic clearance of ilaprazole has not been investigated or has been underestimated. In this study, comprehensive profiling of ilaprazole metabolites (Fig. 1 and 2, A and B; Supplemental Fig. 2) and ilaprazole sulfide metabolites (Fig. 2, C and D) in HLMs was conducted using LC/UV/HRMS. In addition to the previously reported metabolites (ilaprazole sulfone, ilaprazole sulfide, and three monooxidation products) (Seo et al., 2012), several new metabolites of ilaprazole, including metabolites of ilaprazole sulfide (M1, M2, M6, M8, and M9), were detected and structurally characterized in HLMs. Moreover, quantitative analysis using LC/UV revealed that ilaprazole sulfide was the most abundant product and ilaprazole sulfide metabolites, M2, M8, and M9, were the major metabolites in the HLM incubation with ilaprazole (Fig. 2, A and B). Additionally, the HLM metabolic stability experiment showed that ilaprazole sulfide at a low concentration was rapidly metabolized in HLMs (Fig. 5A). The results demonstrated that sulfoxide reduction is the dominant metabolic pathway of ilaprazole in HLMs (Fig. 1), while the formation of other ilaprazole metabolites, ilaprazole sulfone and monooxidation products (M4, M5, and M7), were minor metabolic pathways.

The role of sulfoxide reduction in the clearance of ilaprazole in humans was further examined by the comprehensive detection and characterization of ilaprazole metabolites in human urine and feces using LC/HRMS and various metabolite data-mining methods, such as mass defect filter. As shown in Fig. 6, all of the ilaprazole metabolites detected and characterized in human urine and feces except for M5 were either ilaprazole sulfide (M12) or its metabolites (M1, M2, M6, M8, M9, and M20). The LC/MS peak areas of these ilaprazole sulfide-related components in the urine or feces samples were either higher than or equivalent to M5 or the parent drug suggesting that ilaprazole sulfidederived metabolites were major drug-related components in human excretes. M5 was a relatively minor metabolite in urine, but was not found in feces. 
It is also important to understand the role of direct elimination of ilaprazole via liver and kidney in the clearance of ilaprazole in humans. Ilaprazole was found in human feces samples, but it was not detected in human urine in the current investigation (Fig. 6), consistent with a previous observation (Zhou et al., 2010). The abundance of ilaprazole in feces was comparable to or lower than ilaprazole sulfide or its metabolites (M1, M2, M6, M8, M9, and M20) based on their peak intensities in the LC/MS profiles (Fig. 6, A and C). Ilaprazole in feces can be unabsorbed ilaprazole and/or ilaprazole excreted from liver. Apparently, the direct elimination of the parent drug via either kidney or liver is not a significant clearance pathway of ilaprazole in humans. Based on the results from in vitro and in vivo biotransformation experiments we propose that sulfoxide reduction to ilaprazole sulfide, followed by its rapid oxidation of ilaprazole sulfide to M8, M9, and M20, is the dominant metabolic clearance pathway of ilaprazole in humans. M8 and M9 undergo further biotransformation to M1, M2, and M6 (Fig. 1). A human radiolabeled absorption, distribution, metabolism, and excretion study or quantitative analysis of ilaprazole and its metabolites in human urine and feces using synthetic standards in the future would to confirm the proposed metabolic clearance pathways of ilaprazole in humans.

Sulfoxide reduction of PPIs has been considered as a nonenzymatic chemical reaction (Setoyama et al., 2006; Ren et al., 2008; de Bortoli et al., 2013). In the current study, ilaprazole sulfide was formed in HLM incubation without NADPH (Fig. 4B), suggesting that P450 is not involved in the sulfoxide reduction. The formation of ilaprazole sulfide in the incubation with deactivated HLMs (preheated at $80^{\circ} \mathrm{C}$ for 10 minutes) and NADPH (Fig. 4D) further confirmed that sulfoxide reduction of ilaprazole in HLMs is mainly a chemical reaction with no or minimal involvement of $\mathrm{P} 450$ or other common metabolizing enzymes in HLMs. Interestingly, ilaprazole was very stable in human plasma (Fig. 5B), which may be associated with the high protein binding of ilaprazole in plasma. In addition, the incubation of ilaprazole in buffer without HLMs only generated about $30 \%$ of ilaprazole sulfide compared with that in HLM incubations (data now shown), which happened in a similar HLM incubation with rabeprazole (Ren et al., 2008). These observations suggest that some unknown nonenzymatic factors in HLMs may enhance the sulfoxide reduction reaction. For example, components in HLMs may increase the solvability of ilaprazole in the incubation medium, resulting in faster ilaprazole reduction. The chemical mechanism of the sulfoxide reduction of PPIs remains to be investigated.

In summary, biotransformation of ilaprazole in HLMs and humans was investigated in this study. The in vitro results suggest that ilaprazole undergoes three primary metabolic pathways in HLMs: sulfoxide reduction to ilaprazole sulfide, sulfoxide oxidation to ilaprazole sulfone, and monooxidation to multiple metabolites. Ilaprazole sulfide is further rapidly converted to multiple oxidative metabolites. Sulfoxide reduction is the major metabolic pathway, while sulfoxide oxidation and monooxidation of ilaprazole are minor metabolic pathways in HLMs. Furthermore, all ilaprazole metabolites found in human urine and feces except for a minor monooxidation product (M5) in urine were ilaprazole sulfide and its metabolites. Collectively, we propose that sulfoxide reduction to ilaprazole sulfide, followed by its further oxidative metabolism, plays a dominant role in the clearance of ilaprazole in humans, while CYP3A-mediated sulfoxide oxidation is a relatively minor metabolic clearance pathway. Sulfoxide reduction of ilaprazole is most likely a nonenzymatic reaction, although its mechanism remains to be investigated. Consequently, we predict that the exposure of ilaprazole to humans will not be significantly affected by a coadministered inhibitor or inductor of CYP3A4, other P450 enzymes, and common phase I and II enzymes as well as polymorphism of these metabolizing enzymes.

\section{Acknowledgments}

We thank Dr. Alicia Du for supporting the research project, Dr. Xiaomei Zhang for providing ilaprazole, and Livzon Pharmaceutical Group Inc. for providing ilaprazole sulfone and ilaprazole sulfide.

\section{Authorship Contributions}

Participated in research design: $\mathrm{Pu}$, Zhu.

Conducted experiments: Pu, Wang.

Performed data analysis: Pu, Tang, Zhu.

Wrote or contributed to the writing of the manuscript: $\mathrm{Pu}$, Zhu.

\section{References}

Calabresi L, Pazzucconi F, Ferrara S, Di Paolo A, Tacca MD, and Sirtori C (2004) Pharmacokinetic interactions between omeprazole/pantoprazole and clarithromycin in health volunteers. Pharmacol Res 49:493-499.

Cao S, Zhou G, Chen Y, Guo D, Tan Z, Fan L, Hu H, Qin X, Zhou H, Ou-Yang DS, et al. (2015) Gender, but not CYP2C19 genotypes and CYP3A phenotypes, is a major determinant of ilaprazole pharmacokinetic. Am J Life Sci 3:14-20.

Cao S, Zhou G, Ou-Yang DS, Wu HZ, Xiao K, Chen Y, Guo D, Fan L, Tan ZR, Hu HT, et al. (2012) Pharmacokinetic interactions between ilaprazole and clarithromycin following ilaprazole, clarithromycin and amoxicillin triple therapy. Acta Pharmacol Sin 33: $1095-1100$.

Cho H, Choi MK, Cho DY, Yeo CW, Jeong HE, Shon JH, Lee JY, Shin JS, Cho M, Kim DY, et al. (2012) Effect of CYP2C19 genetic polymorphism on pharmacokinetics and pharmacodynamics of a new proton pump inhibitor, ilaprazole. J Clin Pharmacol 52:976-984.

de Bortoli N, Martinucci I, Giacchino M, Blandizzi C, Marchi S, Savarino V, and Savarino E (2013) The pharmacokinetics of ilaprazole for gastro-esophageal reflux treatment. Expert Opin Drug Metab Toxicol 9:1361-1369.

Dent J, El-Serag HB, Wallander MA, and Johansson S (2005) Epidemiology of gastro-oesophageal reflux disease: a systematic review. Gut 54:710-717.

Fenneteau F, Poulin P, and Nekka F (2010) Physiologically based predictions of the impact of inhibition of intestinal and hepatic metabolism on human pharmacokinetics of CYP3A substrates. J Pharm Sci 99:486-514.

Hisaka A, Kusama M, Ohno Y, Sugiyama Y, and Suzuki H (2009) A proposal for a pharmacokinetic interaction significance classification system (PISCS) based on predicted drug exposure changes and its potential application to alert classifications in product labelling. Clin Pharmacokinet 48:653-666.

Hunfeld NG, Mathot RA, Touw DJ, van Schaik RH, Mulder PG, Franck PF, Kuipers EJ, and Geus WP (2008) Effect of CYP2C19*2 and *17 mutations on pharmacodynamics and kinetics of proton pump inhibitors in Caucasians. Br J Clin Pharmacol 65:752-760.

Klotz U (2006) Clinical impact of CYP2C19 polymorphism on the action of proton pump inhibitors: a review of a special problem. Int J Clin Pharmacol Ther 44:297-302.

Klotz U, Schwab M, and Treiber G (2004) CYP2C19 polymorphism and proton pump inhibitors. Basic Clin Pharmacol Toxicol 95:2-8.

Li Y, Zhang W, Guo D, Zhou G, Zhou H, and Xiao Z (2008) Pharmacokinetics of the new proton pump inhibitor ilaprazole in Chinese healthy subjects in relation to CYP3A5 and CYP2C19 genotypes. Clin Chim Acta 391:60-67.

Li Z, Zou D, Ma X, Chen J, Shi X, Gong Y, Man X, Gao L, Zhao Y, Wang R, et al. (2010) Epidemiology of peptic ulcer disease: endoscopic results of the systematic investigation of gastrointestinal disease in China. Am J Gastroenterol 105:2570-2577.

Ma S and Chowdhury SK (2013) Data acquisition and data mining techniques for metabolite identification using LC coupled to high-resolution MS. Bioanalysis 5:1285-1297.

Mainz D, Borner K, Koeppe P, Kotwas J, and Lode H (2002) Pharmacokinetics of lansoprazole, amoxicillin and clarithromycin after simultaneous and single administration. $J$ Antimicrob Chemother 50:699-706.

Myung SW, Min HK, Jin C, Kim M, Lee SM, Chung GJ, Park SJ, Kim DY, and Cho HW (1999) Identification of IY81149 and its metabolites in the rat plasma using the on-line HPLC/ESI mass spectrometry. Arch Pharm Res 22:189-193.

Park GJ, Bae SH, Park WS, Han S, Park MH, Shin SH, Shin YG, and Yim DS (2017) Drug-drug interaction of microdose and regular-dose omeprazole with a CYP2C19 inhibitor and inducer. Drug Des Devel Ther 11:1043-1053.

Periclou AP, Goldwater R, Lee SM, Park DW, Kim DY, Cho KD, Boileau F, and Jung WT (2000) A comparative pharmacodynamic study of IY- 81149 versus omeprazole in patients with gastroesophageal reflux disease. Clin Pharmacol Ther 68:304-311.

Qiao HL, Hu YR, Tian X, Jia LJ, Gao N, Zhang LR, and Guo YZ (2006) Pharmacokinetics of three proton pump inhibitors in Chinese subjects in relation to the CYP2C19 genotype. Eur J Clin Pharmacol 62:107-112.

Ren S, Park MJ, Kim A, and Lee BJ (2008) In vitro metabolic stability of moisture-sensitive rabeprazole in human liver microsomes and its modulation by pharmaceutical excipients. Arch Pharm Res 31:406-413.

Ruan Q, Peterman S, Szewc MA, Ma L, Cui D, Humphreys WG, and Zhu M (2008) An integrated method for metabolite detection and identification using a linear ion trap/Orbitrap mass spectrometer and multiple data processing techniques: application to indinavir metabolite detection. $J$ Mass Spectrom 43:251-261.

Savarino E, Ottonello A, Martinucci I, Dulbecco P, and Savarino V (2016) Ilaprazole for the treatment of gastro-esophageal reflux. Expert Opin Pharmacother 17:2107-2113.

Seo KA, Lee SJ, Kim KB, Bae SK, Liu KH, Kim DH, and Shin JG (2012) Ilaprazole, a new proton pump inhibitor, is primarily metabolized to ilaprazole sulfone by CYP3A4 and 3A5. Xenobiotica 42:278-284.

Setoyama T, Drijfhout WJ, van de Merbel NC, Humphries TJ, and Hasegawa J (2006) Mass balance study of $[14 \mathrm{C}]$ rabeprazole following oral administration in healthy subjects. Int $J$ Clin Pharmacol Ther 44:557-565.

Shimizu M, Uno T, Yasui-Furukori N, Sugawara K, and Tateishi T (2006) Effects of clarithromycin and verapamil on rabeprazole pharmacokinetics between CYP2C19 genotypes. Eur J Clin Pharmacol 62:597-603. 
Shin JS, Lee JY, Cho KH, Park HL, Kukulka M, Wu JT, Kim DY, and Park SH (2014) The pharmacokinetics, pharmacodynamics and safety of oral doses of ilaprazole 10, 20 and $40 \mathrm{~ms}$ and esomeprazole $40 \mathrm{mg}$ in healthy subjects: a randomised, open-label crossover study. Aliment Pharmacol Ther 40:548-561.

Ushiama H, Echizen H, Nachi S, and Ohnishi A (2002) Dose-dependent inhibition of CYP3A activity by clarithromycin during Helicobacter pylori eradication therapy assessed by changes in plasma lansoprazole levels and partial cortisol clearance to $6 \beta$-hydroxycortisol. Clin Pharmacol Ther 72:33-43.

Wang H, Ou N, Lang L, Shi R, Hu P, and Jiang J (2016) Pharmacokinetics and pharmacodynamics of intravenous ilaprazole in healthy subjects after single ascending doses. Xenobiotica 46: $1133-1141$.

Xuan JW, Song RL, Xu GX, Lu WQ, Lu YJ, and Liu Z (2016) Modeling the cost-effectiveness of ilaprazole versus omeprazole for the treatment of newly diagnosed duodenal ulcer patients in China. J Med Econ 19:1056-1060.

Xue Y, Qin X, Zhou L, Lin S, Wang L, Hu H, and Xia J (2016) A randomized, double-blind, active-controlled, multi-center study of ilaprazole in the treatment of reflux esophagitis. Clin Drug Investig 36:985-992.

Yasuda S, Horai Y, Tomono Y, Nakai H, Yamato C, Manabe K, Kobayashi K, Chiba K, and Ishizaki T (1995) Comparison of the kinetic disposition and metabolism of E3810, a new proton pump inhibitor, and omeprazole in relation to $S$-mephenytoin $4^{\prime}$-hydroxylation status. Clin Pharmacol Ther 58:143-154.

Zhou G, Shi S, Zhang W, Tan Z, Chen Y, Guo D, Zhou H, Hu H, and Tan J (2010) Identification of ilaprazole metabolites in human urine by HPLC-ESI-MS/MS and HPLC-NMR experiments. Biomed Chromatogr 24:1130-1135.

Zhou G, Tan ZR, Zhang W, Ou-Yang DS, Chen Y, Guo D, Liu YZ, Fan L, and Deng HW (2009) An improved LC-MS/MS method for quantitative determination of ilaprazole and its metabolites in human plasma and its application to a pharmacokinetic study. Acta Pharmacol Sin 30: 1330-1336.

Zhu M, Ma L, Zhang D, Ray K, Zhao W, Humphreys WG, Skiles G, Sanders M, and Zhang H (2006) Detection and characterization of metabolites in biological matrices using mass defect filtering of liquid chromatography/high resolution mass spectrometry data. Drug Metab Dispos 34:1722-1733.

Address correspondence to: Dr. Mingshe Zhu, MassDefect Technologies, PO Box 172, Rocky Hill, NJ 08553. E-mail: mingshe.zhu@yahoo.com 